psychological well-being at 30 months did not differ between ICD and placebo treatment groups. Physical functioning (assessed by the Duke Activity Status Index) did not differ between the groups throughout the study, and amiodarone therapy had no discernable effect on quality of life.

In the second paper, Poole et al. looked at the long-term, prognostic significance of ICD shocks among patients enrolled in SCDHeFT. During follow-up (median 45.5 months) a shock was experienced by 269 of the 811 individuals who had an ICD. The shock was appropriately triggered (by ventricular tachycardia or ventricular fibrillation) in $47.6 \%$ of these patients and inappropriately triggered in $32.3 \%$. A total of 182 patients with an ICD died during the study; the most common cause of death was progressive heart failure (42.9\%). Multivariate analysis showed that an appropriate ICD shock increased the risk of death by more than five times (hazard ratio $5.68,95 \% \mathrm{Cl}$ 3.97-8.10, $P<0.001)$, whereas an inappropriate shock almost doubled the mortality risk (hazard ratio $1.98,95 \% \mathrm{Cl} 1.29-3.05, P=0.002)$. Future research efforts could be directed towards the identification of therapeutic options to improve the prognosis of patients with heart failure who receive an ICD for primary prevention.

Original articles Mark DB et al. (2008) Quality of life with defibrillator therapy or amiodarone in heart failure. $N$ Engl J Med 359: 999-1008

Poole JE et al. (2008) Prognostic importance of defibrillator shocks in patients with heart failure. N Engl J Med 359: 1009-1017

\section{Out-of-hospital cardiac arrest: validated criteria for terminating resuscitation attempts}

Survival among patients who suffer an out-ofhospital cardiac arrest $(\mathrm{OHCA})$ varies widely and often depends on whether resuscitation at the scene is successful. Identification of individuals unlikely to benefit from continued in-hospital resuscitation attempts could decrease resource use, reduce the costs and risks associated with emergency transport, and allow medical personnel to focus on patients who are most likely to survive.

Sasson et al. have retrospectively validated the basic life support (BLS) and advanced life support (ALS) rules used to identify patients with OHCA who are unlikely to benefit from in-hospital resuscitation. After an appropriate resuscitation effort at the scene, if the patient does not meet either the BLS or ALS criteria, resuscitation attempts should cease.

Data were analyzed from 5,505 patients who were treated for OHCA and included in the Cardiac Arrest Registry to Enhance Survival. Overall, $17.2 \%$ of OHCA patients were pronounced dead at the scene on the basis of standard protocols. Out-of-hospital resuscitation attempts would have been terminated in $47.1 \%$ and $21.7 \%$ of patients had the BLS or ALS rule, respectively, been applied. Although the BLS rule misclassified five individuals who would have met the criteria but actually survived to hospital discharge, it had an acceptable positive predictive value of $99.8 \%$. None of the patients who met the ALS criteria survived and this rule, therefore, had a positive predictive value of $100 \%$. The authors call for a prospective study to assess the use of BLS and ALS rules in clinical practice.

Original article Sasson C et al. (2008) Prehospital termination of resuscitation in cases of refractory out-of-hospital cardiac arrest. JAMA 300: 1432-1438

\section{False lumen size: a powerful predictor of acute type B aortic dissection}

Mortality and morbidity from aortic dissection remain unacceptably high. Early complications carry a high risk of death, and as the anatomy of aortic dissection is usually complex, total aortic diameter alone cannot predict their development.

Chang et al. have used CT to identify predictors of in-hospital complications including rupture, progression and malperfusion syndrome in patients with Standford type B aortic dissection. In total, 55 consecutive patients were examined by cross-sectional CT (51 men; mean age 58.3 years). Patients were classified according to whether they had in-hospital complications $(n=24)$ or a stable hospital course $(n=31)$.

On multivariate analysis, complications were independently predicted only by the number of involved branch vessels $(P=0.004)$ and maximal false lumen area $(P=0.02)$. The frequency of in-hospital complications was significantly raised in patients with two or more 